\title{
Analytical indices
}

\author{
(A) Index of genres \\ An entry in square brackets indicates approximation to the genre. \\ Poem number followed where appropriate by line number.
}

Ballad 116(B); 147; 148; 149; 150

(more a lyric); 151; 152; 153; $154 ; 155 ; 156 ; 157 ; 158 ; 159$; $238 ; 259$

Biblical narrative and paraphrase (see also Psalms) 178; 202; 231; $249 ; 255 / 67-81$

Chorography $163 ; 186 / 1-88$; [190/89-154]; 261

Country-house poem [137]; 191; 192; 234; 237; [244]; [247]; [276]

Dedicatory/complimentary poem $174 ; 271$

\section{Dialogue}

love-dialogue, wooing dialogue 3 ; 36; 73; 101; 134; 189/47-74; 229; 240(B); 260

other dialogue $1 ; 6 ; 19 ; 26 ; 28 ; 40$; $41 ; 67 ; 139 ; 145 ; 205 ; 208 ; 241$; $242 ; 246 ; 250 ; 262 ; 268 ; 273$

\section{Drama}

chorus from $33 ; 34$

song from $93(\mathrm{~B}) ; 94 ; 175 ; 193$; $195 / 1-10,55-70 ; 211 ; 214 ; 218$ other extract $93 ; 193 ; 195 ; 240$; 253

Echo device 91
Eclogues $4 ; 6 ; 7 ; 8 ; 9 ; 15 ; 16 ; 17 ; 25 ; 26$; $27 ; 28 ; 32 ; 38 ; 39 ; 40 ; 64 ; 67 ; 80$; $83 ; 141 ; 160 ; 161 ; 162 ; 169 ; 196$; $215 ; 262 ; 271$

allusive/allegorical 42; 43; 62; 129; $138 ; 139 ; 140 ; 146 ; 183 ; 184$; $185 ; 199 ; 201 ; 202 ; 204 ; 206$; $226 ; 227 ; 228 ; 230 ; 239 ; 241$; $242 ; 250 ; 252 ; 268 ; 275 ; 277$ amoebean $1 ; 46 ; 49 ; 51 ; 139 ; 145$; $166 ; 169 / 137-78 ; 202 ; 219$; 227/9-38

from eclogue series $38 ; 40 ; 41 ; 57$; $58 ; 59 ; 83$

piscatory 219

Elegy (for the dead) or other mourning-poem $5 ; 43 ; 52 ; 57$; 58; 138; 147; 148; 152; 199; 201; $230 ; 252 ; 259 ; 272 ; 277$

Emblem/motto at close $1 ; 2 ; 38 ; 40$; $41 ; 141 ; 169$

Epigram 13; 21; 130; 143; 170; 248

Epistles: see Letters

Epode (after Horace) 12; 225; 232

Eulogy $6 ; 27 ; 38 ; 63 ; 64 ; 65 ; 130 ; 137$; $138 ; 139 ; 146$

Fable 83/31ff.; 206/145-220 
Folk song or imitation 24; 29; 30 (See also 'Song')

Georgic 10; 11

Hymn to pastoral/classical gods 175 ; 193

Hymn (Christian) 187; 188

Idyll (Theocritus) $1 ; 2 ; 3 ; 4$

Letter or verse epistle [13]; 59; [109]; 164/37-84; [220]; 223; 245; 247

Lyric (see also Song) 67; 78; 84; 85; 86; 89; 90; 91; 101; 107; 108; 112; $113 ; 114 ; 115 ; 116 ; 117 ; 118$; $119 ; 120 ; 123 ; 124 ; 126 ; 127$; $128 ; 134 ; 135 ; 136 ; 150 ; 170$; $171 ; 179 ; 209 ; 216 ; 222 ; 229$; $235 ; 236 ; 243 ; 246 ; 248 ; 251$; $260 ; 263 ; 272 ; 273 ; 274 ; 276$

Mythological tale 61; 66; 92; 94

Narrative $31 ; 90 ; 164 ; 210$

'Nymphal' 166; 167

Ode or ode-like form 22; 71; 77; 90; 91 (canzon); 102; 146; 217; 224; 231

Pageant/entertainment/celebration $46 ; 63 ; 65 ; 66 ; 137 ; 182$

Pastourelle 30

Prefatory poem 174, 271

Prose narrative, verse from $22 ; 23$; $24 ; 47 ; 48 ; 49 ; 50 ; 51 ; 52 ; 63$; $71 ; 72 ; 73 ; 74 ; 75 ; 76 ; 77 ; 79$; $80 ; 99 ; 105 ; 131 ; 144 ; 176 ; 210$; 221
Prose treatise, verse from $14 ; 35$

Psalm 87; 111

Quantitative verse 6; 50; 61; 100; 106

Riddle 49/141ff.; 125

Sestina (double) 51

Song embedded in eclogue 38/37ff.; 83/31ff.; 100; 160/127ff., 165ff.; 161/123ff.; 162/85ff., 145ff., 193ff.; 164/165ff.; 168/33ff.; 169/137ff.; 183/19ff.; 189/47-74, 235-62 (in verse narrative); 207 ; 208; 215/16-43, 64-80 (verses); 239/229-302

Song, other (see also Folk song, Lyric) $69 ; 70 ; 133 ; 144 ; 170 ; 172 ; 173$; $194 ; 205 ; 241 ; 242 ; 254 ; 264$; $265 ; 267 ; 269 ; 270$

Sonnet $81 ; 82 ; 97 ; 98 ; 103 ; 104 ; 109$; $110 ; 125 ; 126 ;$ [176]; 181; 197; 198

Translation $1 ; 2 ; 3 ; 4 ; 5 ; 6 ; 7 ; 8 ; 9 ; 10$; $11 ; 12 ; 13 ; 14 ; 15 ; 16 ; 17 ; 20 ; 21$; $22 ; 23 ; 24 ; 33 ; 34 ; 35 ; 36 ; 37 ; 57$; $59 ; 170 ; 171 ; 176 ; 177 ; 203$

Verse narrative, extract from $44 ; 45$; $55 ; 56 ; 92 ; 95 ; 96 ; 100 ; 121 ; 122$; $142 ; 168 ; 186 ; 189 ; 190 ; 206$

Verse treatise, extract from 249

Wooing-speech $2 ; 7 ; 84 ; 86 ; 95$; $99 ; 116 / 21-8 ; 120 ; 126 ; 154$; $155 / 25-48 ; 169 / 33-112$, $137-78 ; 171 ; 216 ; 276$

\section{(B) Analytical index of themes}

\section{Poem number followed where appropriate by line number.}

The span of themes and issues treated in these poems is wide-ranging and complex. It seemed best to present them analytically rather than in a single alphabetical list, so that related issues might appear together as much as possible. The first group of general themes and motifs is followed by eight others: 
Shepherds' lives

Court and country, simple and complex man

Love

Nature

Golden Age/Paradise

Allegory/allusion/didacticism

Myth and the supernatural

Religious and moral themes

\section{General themes and motifs}

Range of subjects in pastoral

25/129-36

'Virgilian cycle': pastoral to epic 25/27-30, 49-76

Pastoral decorum: humble style

25/80-88, 128; 40/65-80; 64/13;

$215 / 64-72 ; 220 / 9-16 ; 243 / 1-10$

Higher themes (paulo maiora) 8;

42/236-47; 64/30-33, 150-2;

161/17-60; 221(B)/8-21;

221(C)/50-56; 230/87

higher wisdom, above pastoral 10/29-42, 49-54; 183/33-68

Praise of shepherds: their venerable lineage: famous shepherds in myth and history $17 / 39-56$; 41/127-52; 100/15-40; 188/1-4; 213(A); 251/1-4

Types of herdsmen (neatherd, shepherd, goatherd etc.) 1 ;

41/1-8

Shepherd vs other occupations

56/78-80; 166

shepherd vs ploughman/farmer $17 / 20-38 ; 100 / 5-14$

shepherd vs forester/hunter 46 ; 166/45-98; 246

shepherd vs fisherman 166/115-68; 219

shepherds and sailors 258

shepherd and 'herdman' 67

Ploughman 19, 259

Sheep vs goats $41 / 1-8 ; 188 / 53 ; 202 / 6$

\section{Shepherds' lives}

Pastoral/rural content $6 / 52-9 ; 10$; 44/10-14, 127-234 passim; 54/9-36; 56/82-95; 69; 75; 78; 93(B); 96/1-14, 113-19; 97/8ff.; 160/19-30; 163(B)/172-82; 168/33-60; 185/161-80; 186/173-242; 206/60-106; 208/8-42; 213(A)/7-10; 217/105-8; 221(A)/120-40; 223/21ff.; 225; 247; 248/1-10; 221(C)/22-42; 224; 236/1-49; 247/1-18; 251/31-8; 253/21-2, 43-6; 255/15-24, 52-7; 261/51-64; 270 under beech (cf. Virgil, Eclogue

I) $4 / 7-10 ; 6 / 1-5 ; 12 / 21-6$; $221(\mathrm{~A}) / 127 ; 274$

happy family life 10/97-100;

12/37-46; 29/57-60; 191/89-98;

217/105-12; 221(A)/122-7;

221(C)/37-40; 237/327-48;

247/157-70; 261/59-64

blessedness (cf. Horace, Epode II)

$10 / 55 f f ; 12 ; 20 / 31-5 ; 69 / 25 ; 225$;

$232 ; 253 / 20$

gods have favoured country/

shepherd life $7 / 60-61 ; 9 / 17-18$;

97/5-6; 204/86-96; 213(A)/1-7;

$219 / 246$

Shepherds' virtue 96/123-6;

186/153-206; 251/1-2;

$253 / 37-54$

humility 17/69-72; 41/9-16, 
93-104; 44/127-234 passim;

78/9-16; 93(B)/5-8; 97/8-12;

100/8-10; 160/19-30;

169/67-72; 186/153ff.;

187/33-6; 190/69-80;

206/27-40; 208/29-42;

215/65-70; 220/1-4; 247/11-16;

253/37-54; 274/43-4

Shepherd lore; shepherd wisdom

10/29-42; 160/41-2; 183/33-70;

206/67-78, 225-32; 226/133-60;

232/11-52; 253/19-32

Shepherds' prosperity $2 / 34-8$;

3/35-6; 7/20-22; 10/3-4,

81-96; 28/83-8; 46/19-21;

55/58-64; 99/13-20; 155/29-36;

194/14-17; 213(A)/67,

86, 126-7; 226/120-21;

252/59-64

contented poverty $10 / 1-24$;

44/116-17; 236/40-42

austere but ample food 12/51-4;

19/1-28 ('sufficiency');

153/93-108; 232/53-74;

261/5-10

shepherds' hospitality 6/79-83,

44/15-23, 100-17, 235-61;

219/284-7

Pleasures of shepherd life 56/82-98;

185/155-82. See also 'Pastoral/

rural content' above.

Hardships of shepherd life: 'real' shepherds and rustics

26/39-80; 28; 55/9-50; $183 / 72-3 ; 256$

complaint against poverty 28 ;

254/33-48; 256; 259; 275

Mourning dead shepherd/

shepherdess 5/26-30; 27; 43;

$52 ; 57 ; 58 ; 148 ; 152$. See also

'Elegy' in Index of genres.

Evil among shepherds or in rural life 26/97-104; 28/41-74
Satire/derogatory treatment of shepherds/rustics 21 ; 264

Discontented/uprooted/wandering shepherd $6 ; 40 / 9-16 ; 141 ; 167$ (satyr)

Comic shepherds 21; 49; 73; 79; 206/126-8; 264; 265; 269

Description of shepherd and dress 56/56-64; 71/59-70; 74/17-42; 149/49-54; 153/9-20, 25-8; 161/177-88, 173a-184a; 162/9-16; 207/21-30; 256/36-8; $269 / 3 ; 275 / 53-4$

Description of shepherdess \& dress 67/39-48; 68/21-36; 71/31-50; 74/43-68; 126/33-8; 161/141-52, 137a-148a; 207/11-20; 267/13-20

Shepherds' tasks 10/81-96;

$11 ; 12 / 7-20,37-46$

(countrywoman); 16/129-52

(all working classes); 28/101-16; 57/24-36; 59/10-20; 95/13-38;

122/95-106; 151/88-95

(shepherdess); 166/185-236;

187 ; 188; 204/173-6; 244

Sheep-farming 163(C)/19-54;

166/185-236; 182

Shepherds' remedies: curing sheep $41 / 85-8 ; 160 / 31-4$

Shepherd as hireling/owner

55/58-64; 115/17-24;

193/67-74; 226/167-70;

254/33-6

Shepherds' feasts and country delicacies 29/7-12, 25-8, 41-4; 56/59-61; 73/1-4; 95/39-48, $55-6 ; 62 ; 113 / 17-18 ; 150 / 24$, 39-42; 153/93-108; 155/37-44; 162/41-8; 163(C)/58-60; 166/221-2; 191/51; 253/51-2; $270 / 33 ; 275 / 49-52$ 
Hunting 12/27-36; 26/143-8;

43/97-168; 44/163-6; 95/19-38;

160/35-6; 163(B)/81-149;

166/45-98; 192/23-36;

193/9-11; 194/20-23;

196/180-5; 228/131-9;

$247 / 25-7 ; 276 / 59-70$

Fishing 166/115-68; 276/25-8

Shepherds' festivals/

sports/'shepherd's holiday'

10/101-10; 43/49-50, 91-6;

49/69-71; 53/7-12; 58/100-9;

59/21-60; 67/38; 91; 96/71-84;

$106 / 22-30 ; 131 ; 136 / 25-32$;

137 ; 150; 151/61-8; 154/79-102;

$155 / 13-24 ; 162 ; 163(\mathrm{C}) / 55-62$;

164/130ff.; 185/23-4;

189/157-367; 193; 195; 207;

$214 ; 228 ; 244 ; 252 / 110-12 ; 254$;

$255 / 25-7 ; 275 / 2$

Lady of May 46; 117/26; 228;

239/72-8; 245

maying 117/25-6; 150; 161/154, 150a; 221(A)/8-11; 228/27-42;

$256 / 33 ; 258 / 9-10$

dances, catalogue of

207/55-62

music 164/133-48

\section{Country sports and games}

26/149-54; 43/43-50, 91-6;

$136 / 29-32 ; 154 / 15-16$;

166/200-1; 228/27-36, 73-8;

245/14-24; 253/53-4

Aristocrat/city-dweller's country

retreat $13 ; 109 ; 192 / 91-106$;

216; 217; 223; 232/54-5; 247;

248. See also 'Country house

Poems' below.

Country house poems 191; 192; 234;

237; 244; [245]

country house prosperity

191/19-44

country house hospitality
191/57-88; 192/47-60; 234;

244/26-44; 245

country house festivities 137;

$192 / 48-60 ; 244 ; 245$

the lord's family 191/83-98

\section{Court and country, simple and} complex man

Pastoral/country vs court/city 16 ; 20/44-5

contrast with courtly disquiet, corruption, opulence, etc 10/5-16, 57-80; 12/3-6, 47-54; 14/6-9; 19/87-98;

20/44-5; 26/131-98; 34/9-12;

42/280-307; 44/127-234

passim; 50/15-28; 54;

56/82-95; 67/53-6; 69;

$75 ; 76 ; 78 ; 93(\mathrm{~A}) / 1-12$;

93(B); 96/85-91, 97/8-12;

113/25-36; 122; 131/107-10;

160/19-30; 162/25-8;

163(B)/172ff.; 163(D)/9-14;

167/137-48; 168/43-4;

185/161-2; 186/207-252;

192/1-16; 208/29-42; 209/1-8;

217/81-108; 221(A)/134-40;

221(C)/8-21; 223; 224; 225;

232/1-12; 236/1-49; 237/321-6;

243/17-26; 245/1-10; 248;

253/45-52; 255/22-4, 52-7,

$64-9 ; 256 ; 274 / 33-68$

such contrast as religious/moral allegory 251

contrast between shepherds' misery and courtiers' prosperity 26/19-30, 43-6, 119-20, 165-82

disillusioned courtier turned shepherd 44/172-89; 76; 78/37-44; 163(B)/163-5 (hermit)

shepherd's disillusionment with court 42/280ff. 
shepherd's wonder at city/court 6/20-26; 42/166-203

Peace: contrast to war $14 / 16-22$; 51/63-4; 146/67-78; 169/1-5; $192 / 67-72 ; 217 / 33-48$; $218 / 57-72 ; 223 ; 261 / 51 \mathrm{ff}$.

Class differences $16 ; 19 ; 26 / 19-30$; 28/35-40; 183/74-5; 204/83-4; $254 / 33-48$

Criticism of/protest against the rich 19/129-45; 26/19-30, 131-82; 28/35-40, 83-96; 259/57-63

Higher orders reliant on shepherd/ rustic 19/1-60

King and shepherd 153

Courtier among shepherds 42/60-97; $44 ; 45 ; 92$

Royal/aristocratic heroine as shepherdess $44 ; 56$; 67 ; 204/83-4; 158/116-35

Shepherdess/country lass as ideal: contrast with court ladies 67/41-52; 68; 93(A)/1-12; 148; $151 ; 185 / 167-8$; 186/207-10; 228/83-92; 247/129-48; 267/33-4

Royal/aristocratic man as shepherd 44 ; 45; 56; 166/233-4; 188/3-4; $216 ; 256 / 30-32 ; 257$

Hermit 163(B)/152-87; 255/15

Love (see also 'Nature' below.)

Nature of love 18/17-24; 80; 145; 160/91-204, 127a-200a; 208; $209 ; 212 / 25-36$

Purer love among shepherds 217/105-12; 228/78-92; 253/37-40; 261/59-64

Transience of love 85

Same-sex love 7; 95; 97; 98; 204

Infatuation in love 15/67-94, 215-26; $38 / 25-8,154-9 ; 67 / 85-120$; $80 / 21-8 ; 210 / 33-56,113-16$
Advice to besotted lover: dialogue with/discourse of older/ wiser shepherd $67 ; 77 ; 80 ; 160$; 204

Evil of love; cruelty or evil in women $15 / 211-14 ; 31 / 49-56$; $76 / 67-72 ; 77 ; 80 / 21-44$, 105-32; 96/90-112; 102/17-22; 106/53ff.; 122/17-20, 45-8; 123; $138 / 52-3 ; 145 ; 160 / 115-50$, $127 \mathrm{a}-150 \mathrm{a} ; 179 / 29-52$; 196/67-108; 269/35-8

Freedom from love 208

General situations/expressions of love 23 ; 24 ; 37; 43/71ff.; 47; 48; $50 ; 51 ; 53 / 1-18 ; 66 ; 67 ; 71 ; 72$; $79 ; 81 ; 82 ; 98 ; 112 ; 114 ; 115$; $116 ; 118 ; 119 ; 121 ; 124 ; 135$; $149 ; 150 ; 157 ; 162 / 85-132$, 145-72, 193-234; 196; 197; 209; $210 ; 219 ; 220 ; 238 ; 263 ; 265$; 277/5-26

General love-narrative $18 ; 31 ; 56 ; 60$; $140 ; 164 ; 210 ; 238$

Changing affections $18 ; 31 ; 40 ; 123$; 138/52-3; 205; 254/57-64; 260

Courtier wooing/seducing shepherdess $30 ; 44 / 25-72$, 262-88; 158; 173

Gods/royalty/noblemen in love with shepherd(esse)s $61 ; 66$; 67/59-68; 97/5-6; 106/11; $176 / 17-28 ; 204 / 85-96$

The Amyntas story 57; 58; 59; 60; 96/101; 106/91

Wooing speech, invitation to love 2; $7 ; 30 ; 73 ; 84 ; 86 ; 95 ; 97 ; 99 ; 101$; $116 / 21-8 ; 117 / 9-18 ; 120 ; 126$; $154 ; 155 / 25-48 ; 169 / 33-112$, $137-78 ; 171 ; 210 / 65-80$, $133-52 ; 216 ; 236 / 64-70$; $270 / 30-44 ; 276$ 
Shepherdess's love-speeches 29;

85; 149; 159; 161/201ff., 197ff.; 164/37-84 (letter); 197; 263/31-60

Love-dialogue $3 ; 30 ; 36 ; 73 ; 101 ; 117$; 134; 189/47-74; 210/163-272; 229; 240(B); 260

Asking for or exchanging kisses 24; 71/87-91; 95/79-84; 117/13-15, $23-4 ; 126 ; 127$; 157/55-66; 229/13-14

Love-letter 59

'Love's feast' 53/9

Other courtship and love-exchanges 40/41-6; 56/54-98; 59;

71/73-102; 72/19-30, 79; 101; $107 ; 117 ; 126 ; 127 ; 131 / 29-58$; 154/25-36, 79-150; 157; 159; 161/123-242, 120a-238a; 207/63-110; 265

Shepherds' love-gifts 2/40-42, 56-9; $7 / 40-55 ; 40 / 43-4 ; 49 / 81-6$; 59/25-38, 45; 60/7-8; 71/95-8; 84/9-20; 85/13-18; 95/15-18, 49-54, 64-75; 98/8-11; 106/53-6; 113/21-4; 134/11-30; 149/33-8; 189/265-355; 216/33-6; 219/223-34; 265/19-20; 269/32-4; $270 / 32-3$

Lover's complaint; unhappiness/ frustration in love $31 ; 32 ; 38$; 40 ; 47; 48; $51 ; 67 ; 70 ; 80 ; 81 ; 89$; $92 ; 102 ; 103 ; 104 ; 106 ; 108 ; 123$; $128 ; 141 ; 142 ; 155 ; 169 ; 176$; 177; 179; 196/43-108; 204; 205; 210/90-112, 133-52, 273-96; $211 ; 233 ; 242 ; 254 / 17-40 ; 260$; $262 ; 263 ; 268 ; 269$

Women's complaint against men 85 ; 92; 145; 149/5-8; 159/96-116; 210/133-52; $263 / 31-60$
Love frustrated by poverty 254/33-48; 260; 262/31-42; 268/53-60

Absence of beloved 51/61-72; 164; 263/1-30

Lover addresses his flocks 23/25-37;

31/61-4; 32/1-2, 53-60; 57/13-49; 102/38-40; 103; $169 / 25-32 ; 210 / 90-104$

Flocks pine in sympathy with shepherd, mourn the dead etc. 57/13-49; 67/97-104; 108/1-2, 17-24, 27; 120/5-8; $129 / 31-48 ; 138 / 28-30,155$, 188-90; 141/10-18; 152/17-20; 169/17-32, 41-2; 196/55-8; 212/3-6

Dying for love 9; 31; 32; 58; 70/7; 81/9; 106/107-9; 147/17-20; 179; 269/14ff.

Lament for dead beloved 57; 58; 148; 152

Willow wreath for despair or mourning in love $31 / 40$; 93(A)/13-15; 123/87-8; 148/2, $74 ; 205 ; 210 / 65-76 ; 269 / 4$

\section{Nature}

Description of Arcadia 105, 106/100-1

Beautiful landscape or setting, springtime scene etc. 10/17-22; 12/21-6; 45/1-45; 50; 63/1-8; $105 ; 106 / 100-2 ; 107 / 1-6 ; 118$; 122/115-18, 131-4; 132/11-20; $136 ; 146 ; 154 ; 160 / 7-16 ; 165$; $178 / 1-24 ; 186 / 21-88 ; 189 / 1-28$; 206/107-20, 185-98; 216/9-16; 218/1-36; 224/13-28; 231/10ff.; 232/75-92; 233; 237/243-84; 247; 255/4-14, 28-48; 276; 277/7-22. See also 'Locus amoenus' below. 
Locus amoenus $2 / 45-8 ; 20 ; 22 / 16-22$;

$40 / 1-8 ; 45 / 1-45 ; 71 / 1-30$;

80/5-6; 86/3-32; 99/37-45;

154/7-10; 171; 189/179-98;

196/1-8; 206/107-16, 185-98;

$211 ; 224 / 25-6 ; 252 / 43-56$;

253/1-12

Other nature-description

163(A)/8-22; 163(C)/7-26;

163(D)/3-8; 166/75-91;

$186 / 1-88 ; 203 ; 255 ; 266$

Landscape (artistically formalized)

$163 ; 186 / 1-82 ; 235$;

237/181-226; 258; 266

Rugged nature as retreat 54

Solitude in nature $50 ; 203 ; 274 / 29-32$

Landscape moralized 237/143-62;

252/73-82; 253

Nature as a spiritual or contemplative power; nature mysticism 232/37-46; 255

Beloved's presence affects/ embellishes landscape 1/41-8; 50/29-42; 57/59-67; 114/1-20; 121/45-76; 136; 162/85-132, 149-64, 205-10; 164/182-280 passim; 194/36-9; 196/127-32, 145-50; 216/29-32; 231/10-14; 237/285-316; 242/11-16, 23-8; 276/39-40, 73-80

Nature responds to poet's/lover's happiness 146; 157/62-7; 277/3-12

Nature affected by poet's unhappy love $102 / 1-30$; 108/25-6; $121 / 76-82 ; 142 ; 196 / 59-60$

Frustrated lover retreats to nature $51 ; 106 / 110-22 ; 159 / 65-8$; 196/52-4

Contrast between happy nature and unhappy shepherd 141/7-12, 181-228; 215/16-43; 232/37-48; 233
Address to nature; pathetic fallacy

5/1-19, 32-5; 23/11-13;

$38 / 73-95 ; 39 / 33-44 ; 50 ; 51$;

52/4-30; 81; 82; 102/1-30; 141;

179/9-16; 211; 215/15ff.

Nature mourns with poet or at general calamity $5 / 23-4,32-5$; 9/12-14; 52/1-30; 57/13-49; 129/97-108; 138/31-2, 74-9, 155-67; 152/17-21; 199/23-31; 212/7-18; 230/39-43; 252/119-50; 263/25-30

Nature responds to birth of Christ 249/79-88

Animals willingly yield/sacrifice themselves $91 / 57-63 ; 164 /$ $250-57 ; 191 / 27-38 ; 234 / 23-8$

Catalogue of flowers 7/45-50;

38/136-44; 39/87-94; 155/73-80; 161/155-7, 151a-3a; 162/15-24 (language of flowers); 165/21-30; 171/6-7; 186/44-50; 206/191-8; 217/119-25; 228/39-41; 230/141-51; 232/37-44; 237/166-72, 285-92

Catalogue of fruits 191/39-44; 237/285-318

Catalogue of birds 95/15-36; 118/13-16; 146/5-10; 154/2-6; 163(B)/39-70; 165/15-20; 189/3-22; 217/113-18; 237/117-25; 247/38-90; 276/59-70

Catalogue of fish 247/101-10

Medicinal herbs 91; 63(B)/182-222

Destruction of forests and nature 163(B); 163(D)/30-54; $167 / 58-124$

\section{Golden Age/Paradise}

Golden Age 8; 10/115-16; 12/2; $14 ; 33 ; 34 ; 65 / 27-36 ; 161 / 0.1$, 
65-116, 61a-112a; 174/21-30;

190/138; 192/50, 63-4; 274/5-8, 13-22

as reign of Saturn 8/5; 10/116;

$192 / 50$

eternal spring 33/7-11; 139/37-42;

165/9-16, 37-44, 73-88;

274/5-24

spontaneous crops $8 / 20-24,34-6$,

$47-8$; 33/3-6; 105/24; 231/45-8 no navigation $8 / 37-46 ; 14 / 13-16$;

$33 / 12-13 ; 34 / 7-8 ; 161 / 93-6$,

$89 \mathrm{a}-92 \mathrm{a}$

wool grows ready-dyed 8/50-54

Silver/Iron ages $221(\mathrm{~A}) / 64-72$

Other early pastoral times 113

Early pastoral times as sexually

chaste $16 / 24-42 ; 34 / 17-26$;

113/9-16; 228/91-2

as innocently promiscuous

33/14-68

Paradise/Earthly Paradise/Eden

40/10; 165 (Elizium); 166; 167;

184/194; 206/117; 231; 237/93,

142, 249-58, 271, 329-34;

239/146-50; 255/67-82

Prelapsarian/redeemed state $40 / 10$;

231/41-2; 237/253-8, 329-32;

$255 / 47$

\section{Allegory/allusion/didacticism \\ Theory of allegorical pastoral}

25/43-6, 83-102; 83/89-94;

$213(\mathrm{~A}) / 34-8$

Allegory and allusion 27; 42; 43;

213(A)/33-8

personal (poet's or others') 4; 6; 42;

$141 ; 142 ; 147 ; 164 ; 178 / 49-64$;

$183 ; 184 ; 185 ; 186 ; 197 ; 198$;

199; 201; 206; 215; 219; 220;

221(A); 226; 238(?); 252; 262;

$268 ; 273 ; 275$

philosophical 132; 186; 255/19-21 moral/religious $186 ; 187 ; 188$; 190/205-22; 206/145-214; 222; 257/13-24

political/historical 9, 38/22-153; $55 ; 56 ; 62 ; 64 ; 129 ; 130 ; 141$;

$142 ; 143 ; 146 / 31-54 ; 147$;

$163(\mathrm{~A}) / 1-6 ; 167 ; 185 ; 212 ; 217$; $239 ; 241 ; 242 ; 261 / 11-84 ; 271$.

See also next entry.

execution of Charles I 246; 250; $259 ; 272 ; 277$

theological 227

ecclesiastical $41 ; 62 ; 202 ; 227 ; 228$;

230/109-31; 239

academic $178 / 60-64 ; 206 ; 219$;

221(A); 226/129-76; 230/23-36, 103-7

praise of rulers/patrons/ eminent

persons $4 / 11-16$; 6/6-10;

8/10-11; 27; 41/213-230;

42/60-67; 43; 64; 91; 129; 137;

138 ; 146; 169/65-88; 191;

$192 ; 193 ; 194 ; 228 / 169-82$;

261/65-84. See also next entry.

praise of Queen Elizabeth

$38 / 33-153$; 39; 42/40-51, 88-93,

135-65, 204-67; 45/208-16; 46;

$55 ; 63 ; 65 ; 66 ; 91 / 52-76 ; 130$;

139 ;

Satire $122 / 1-72 ; 143 ; 164 / 348-79$;

167/109-20, 137-48; 185;

190/9-68, 211-30; 213(B);

221(A)/99-105

Complaint against the times

28/61-70; 34/27-36;

41/169-206; 183/73-6; 184;

185 ; 190/9-68; 192/67-90;

206/32-48; 206/247-68;

221(A)/71-91; 228/1-22;

238/1-40, 197-204; 239; 259;

$274 ; 275$

Moral themes in pastoral 25/95-9,

$83 ; 184 ; 186 ; 187 ; 188$ 
Local history 163(A), 191/19-26

Shepherd(ess) as ruler $38 / 33-153$;

$52 / 1,128 ; 65$; $91 / 48-76$;

96/22-63; 118; 130; 132/1-8;

146/92-6; 147; 213(A)/164;

166/75, 93-4 (forester), 213-14;

193/13-15; 194/43; 213(A)/164;

236/49ff.; 241/44; 250/25ff.;

253/13-15; 256/31; 272

Shepherd(ess) as courtier 43/25-96;

52; 96/120; 141; 142; 143;

256/32; 257

as 'leader of the flock' (shepherd?

bell-wether?) 129/55-66

Shepherd as country lord 137

Shepherd as warrior $43 / 97-168$; 64;

$96 / 122 ; 218 / 57-72$

Shepherd as scholar; academic

allegory $62 ; 96 / 121 ; 178 / 61 ; 206$;

219(?), 219/282; 230/23ff., 64ff.

Shepherd as poet $28 ; 38 ; 40 / 33-96$;

77/16; 80; 138/50; 183; 184;

186/97-268; 215/44-64;

221(A)/1-63; 252/51-66;

268/79-108

shepherd-singer affecting nature:

Orpheus figure 5/47-50;

42/19-31; 91/17-20; 138/173-5;

201/6-16; 202/187-92;

[214/15]; 226/33-4; 230/42-4

'poets' paradise' 165/101-4

master shepherd/poet 40/81-2, 226

'poet-shepherd-king' (David)

$221(\mathrm{C}) / 23$

shepherd designates 'heir' in

singing 7/36-9; 168/61-84;

226/11ff.

advice by fellow poet/shepherd

$184 ; 185 ; 204 ; 205$

rivalry/comparison bet. shepherds

and singing gods $5 / 56-7$;

8/69-70

earlier pastoral poets 25 poets of old generally $5 / 72-5$;

25/1-18; 77; 161/41-8, 5a-8a;

190/116-38; 206/225-32

decline in poetry $161 / 61-4,1 a-60 a$

frustrated in love, hence lacking

inspiration 40/46-8, 97-112;

45/122; 138/43-53; 204/15-22,

195-202

breaks/throws away pipe in

frustration/grief 38/14-15;

$138 / 27,85,197 ; 141 / 19 ; 152 / 23$;

226/9-10; 228/171; 238/63;

$263 / 13-15 ; 269 / 11$

poetry impaired by poverty and neglect 138/43-53; 184/49-96;

185/105-182; 221(A)/71-98;

226

poets, contemporary/community

5/87-104; 96/99-102, 164; 183;

$184 ; 185 ; 219 ; 226$

Song-contests $1 ; 4$

pledging stakes $1 / 13-24 ; 4 / 1-6$;

49/29-48

contest ends in decisive judgment $1 / 82-7$

honours divided 219/268-83

Other rewards for telling a tale

15/27-48

Shepherd as priest $27 ; 41 ; 88$;

190/53-4; 213(A)/164;

230/113-29; 238; 239

their corruption and neglect of duties 28/67-8; 41/169-204; 65/21-6; 190/53-4; 227/39-40; 230/113-31; 239/9-26

Shepherd as devotee or worshipper $222 / 3 \mathrm{ff}$.

Myth and the supernatural

Metamorphosis 43/199-216;

49/57-62; 58/57-75; 61; 66; 94;

$131 / 2-8 ; 219 / 66-7,165,206-10$;

$220 / 25-8$ 
Worship of pastoral gods 175; 195/22ff.; 193; 194. See also 'Shepherds' Festivals' above.

Muses, Graces, wood-gods, nymphs, fairies See Index of mythological names and allusions.

Making of pan-pipe/syrinx 61/30-39; 94/25-30; 131/1-8

Hyacinth: marks of mourning 5/5-6; $52 / 29 ; 196 / 156$

Dreams 119

Pan as God/Christ 41/49; 160/24; 239/67, 151; 227; 239/67, $151-4 ; 262 / 1 ; 268 / 26(?)$; $271 / 36$

Pan as monarch 146/92, 193; 185/42; 194; 212/17ff.; 226/37-52; $228 / 97 ; 241(\mathrm{~A}) / 42 ; 241(\mathrm{~B}) / 43$; $246 ; 250 ; 268 / 26 ; 271 / 36$; $275 / 5-32 ; 277 / 28-40$
Religious and moral themes (See also 'Allegory/allusion/didacticism' > 'moral/religious' above.) God/Christ as shepherd 17/69; $35 ; 87 ; 110 ; 111 ; 130 / 6 ; 133 / 33$; $178 / 28-32 ; 180 ; 181 ; 187 / 17$; $188 / 45 ; 194 / 46-66 ; 202 / 85-6$; 213(A)/23, 149-62; 213(B)/196; 221(A)/141-54; 221(A)/141-8, 221(C)/43-9; 249/126; 251/45

Lamb of God 35/69-76; 41/53-6; 249/126; 251/48-50

Nativity shepherds $17 / 57-68$; 100/43-7; 133; 188/9-12; 213(A)/129-32; [241]; 249; 251

\section{Religious themes}

vision, exaltation $17 ; 200 ; 237 / 57-76$ homily 35 moral reading of shepherd's tasks $187 ; 188$ religious satire $65 / 21-8 ; 239$ narrative 178,180

\section{Contemplation in/of nature}

50/1-11; 223/23-54; 232/11-52; 253/19-32; 255/49ff.

(C) Pastoral and other fictional names

Pastoral names of real persons are listed below. Their actual names are listed under 'Historical Names'.

Poem number followed where appropriate by line number. Speech-headings are cited only at the point of first occurrence.

Aegon 59/25 (Egon); 98/12; 226/55

Aglaia 121/9ff.; 122/75 ff.

Aglaura 42/204

Alcon 196/183; 199/2ff.

Alcydon/Alcidon 201/65; 203/ 141

Alexis 7/1ff.; 185/0, 1ff.; 189/165;

197/1ff.; 198/1ff.; 201/28;

227/6ff., 82

Alope 93(B) /19
Alpheus 12/61

Alphus 15/1ff.; 17/1ff. Amargana 136/0, 5ff.

Amarillis/Amaryllis 4 (Virgil)/10 (Amaryll); 6/5, 31, 37; 7/15ff.; 58/96; 70/1; 106/50ff.; 123/55ff. (Amarill); 143/12; 145/0, 5ff.; 157/0, 3ff.; 196/11ff.; 226/57ff.; 227/75; 230/67; 241/3ff.; 242/8ff.; $271 / 44$ 
Amintas/Amyntas 7/35ff.; 9/37ff.;

36/2ff.; 57/1ff.; 58/2ff.; 59/2ff.; 60/2ff.; 83/2ff.; 96/101; 106/91; 123/2ff.; 152/38; 154/31, 94; 157/51; 169/132ff.; 199/102; 227/79; 233/21; 241/1ff.; 263/2ff., 16

Amphrisa 93(A)/0

Anander 168/84ff.

Anarchus 239/0, 217ff.

Anbrie 162/67

Arion 202/192

Arodeame 210/298

Ascopart (Ascleparte) 62/22

Astraea 139; 141

Astrophel 43/10ff.; 91/4ff.; 96/100ff.; $114 / 0$

Atlanta 154/100

Batte 160/0, 1ff.; 162/77ff.

Bavius 221/78; 226/42

Bembus 55/9ff.

Betty/Bess 254/30, 67

Bevis 62/21

Bilkin 253/0

Bion 5/1ff.

Bonnyboots 204/11ff.

Borrill 160/0, 1ff.

Calidore 44/6ff.; 45/109ff.

Caridon 59/41

Carill[i] o 24/2

Carimell 204/68ff.

Carmela 73/1ff.

Cassemen 161/124ff., 120a

Caucus 131/24

Chanticleer 161/163, 159a

Chlorin 236/64

Chloris/Cloris 38/122; 103/13;

104/1ff.; 131/55; 146/1; 157/51; 196/91; 199/66; 233/21; 260/5ff.; $262 / 3 ; 263 / 1 \mathrm{ff}$.

Chores 39/76

Cilrana $154 / 81$

Claia 167/21ff.
Claius (Clayes) 154/26

Clarida 131/17

Clitias 59/35

Cloden 131/53

Clora 71/47; 271/45

Clorinda 43/229

Cloris: see Chloris

Clotten 273/1ff.

Codrus 28/1ff.; 262/1ff.

Coladine 131/53

Colin (Clout) 38/0, 21ff.; 40/0, 1ff.; 42/16ff.; 45/103ff.; 55/23 (Colon); 83/0; 91/48; 146/1ff., 106; 96/99; 138/41, 85, 135, 204; 156/9ff.; 157/0, 9ff.; 161/232, 228a; 164/145; 168/61ff.; 190/170ff.; 220/10ff.; 221/85; 265/1ff.; 96/99; 138/41ff.; 228/1ff. (Collen)

Colliden 169/1ff.

Comma 55/23

Corbilus 167/1ff.

Corima $77 / 51$

Corin 31/0, 5ff., 57, 93; 104/3ff.

Corina $70 / 4$

Cornix 16/3ff.; 26/1ff.; 27/65ff.

Coridon/Corydon 7/1ff.; 26/1ff.;

27/1ff.; 42/10; 44/50, 99;

59/41(Caridon); 71/56ff.; 74/9,

56; 77/28; 79/0; 80/1ff.; 89/5ff.; 107/7ff.; 108/35; 112/3ff.; 114/0, 36; 115/3ff.; 116/0, 24; 117/0, 6ff.; 118/9 (Choridon); 120/0, 33; 131/55; 134/0, 1ff.; 147/56.1; 149/0, 3ff.; 154/32, 84; 157/53; 159/83; 196/178ff.; 206/78ff.; 208/4ff.; 220/20; 226/168ff.; 227/1ff.; 250/1ff.; 260/11; 268/0, 1ff.; 275/0, 1ff.; 277/0, 1ff.

Cosma 49/10ff., 38, 79ff.; 132/20ff.

Cuddy 42/166ff.; 138/55, 192ff.; 205/5ff.

Curan 56/19ff. 
Cusse 162/66

Cut (dog) 269/43

Cuttie 183/1ff.; 185/241

Cynthia (Synthia) 126/43

Cytheris 9/0

Daffadil 162/82ff.

Damian 83/1ff.

Damoetas/Dametas 7/36ff.; 32/4ff.; $98 / 12 ; 106 / 26 ; 135 / 0 ; 230 /$ 36

Damon 37/3; 78/3; 82/16; 100/0; 196/1ff.; 198/1ff.; 199/5ff.; 201/0, 5ff.; 226/2ff.; 250/25ff.; 252/0, 1ff.; 262/1ff.; 271/1ff. (Daman); 272/1

\section{Daniel 155/21}

Daphne 1/43, 72; 36/1ff.; 58/27; 66/0; 67/9ff.; 68/31; 106/41-2; 145/19; $148 / 118 ; 202 / 131$

Daphnis 1, 0/1ff.; 3, 0/1ff.; 7/27; 9/0; 135/31ff.; 196/175; 219/0, 16ff.; 227/5; 228/170ff.; 252/0, $24 \mathrm{ff}$.

Diana $22 / 0 ; 23 / 0$

Dick 153/9; 155/19; 254/1ff.

Dickeye 204/1ff.

Dicus 49/27

Doll 155/89ff.

Dorcas 49/8ff.

Doricles 206/13ff.; 208/2ff.

Doridon 189/27ff.

Dorilus 164/1ff.

Doris 202/124ff.

Doron 72/0; 73/1ff.

Dorus 197/8; 271/1ff.

Dory $189 / 127$

Dowsabell 161/0.4, 126ff.

Driades 59/30

Driope 146/1

Duddy 189/164

Dulcina 149/0, 5ff.; 159/1ff.

Dymnus 206/40ff.; 208/3ff.

Eclecta 178/54ff.

Eglantine 131/54
Egliset 178/41

Egon: see Aegon

Eliza 38/34ff.; 39/9ff.; 91/49ff.

Epizenes 55/51

Era $37 / 16$

Ergasto 83/1ff.

Erycine 197

Espilus 46/1ff.

Eubulus 141/1ff.

Faustulus 59/41

Faustus 16

Felicia 167/65ff.

Felisa 106/30

Ferin 131/54

Fida $189 / 47 \mathrm{ff}$.

Flaccus 232/1ff.

Flora 196/103ff./ 202/27ff.

Fusca 220/5ff.

Galatea 5/31; 106/39; 226/58.ff./ $227 / 4 ; 268 / 63 \mathrm{ff}$.

Galbula 17/1ff.

Ganymede 97/1; 98/4ff.

Gemma 221(A)/38

Gill 164/139; 207/35; 259/7

Gillian 153/0, 87ff.

Glaucus 219/217ff.

Glicery 59/37

Gorbo 161/0, 17ff.; 162/79ff.

Gouldy-Locks 162/64

Guerim 215/9ff.

Halcius 166/111ff.

Harpelus 31/0, 3ff.; 179/1ff. (Harpalus)

Hay 240(A)/4ff.

Henn 240(A)/1ff.

Hermes 202/0.3, 6ff.

Hobbinoll 38/0, 1ff.; 40/0, 1ff.; 42/15ff.; 143/1; 189/164

Hyalus 106/4

Hylas 252/65

Hylax (dog) 202/138

Idea $162 / 179$

Idmon 199/4ff. 
Innocence $240 \mathrm{~B}$

Iolas $7 / 57$

Isenbras $161 / 125$

Jack 254/1ff.; 259/0, 7ff.; 212/0, 6ff.

Janus 15/1ff.

Jeffrey $62 / 1$

Joan 161/245, 241a; 239/298; 254/27; 256/21; 269/23 (Jone)

Kala $21 / 1 ; 196 / 141$

Kate 207/38; 269/24

Kit 207/38

Klaius 51/7ff.

Lalus 49/14ff.

Lampur (dog) 1/65

Laurinella 169/8ff.

Lettice $162 / 65$

Leuca 49/76ff.

Lightfoot (dog) 270/19

Linus 206/31ff.; 208/6; 221/75ff.

Lucasia 273/0,1ff.

Luciscus 232/54

Lycanthe (dog) 252/170

Lycaon 202/0.1, 6ff.

Lycidas/Licidas 5/98; 106/27ff.; 230/8ff.

Lycisca $(\operatorname{dog}) 57 / 33$

Lycoris/Licoris 9/0, 2, 42; 227/81

Lysis 201/18ff.

Makyne 18/3ff.

Malkin 173/7

Marina 207/1ff.

Mary 254/28

Meg 254/26

Melampo (dog) 266/36

Melanthus 166/177ff.

Meleager (Meliager) 154/99

Melibeus 4/1; 6/0, 1ff.; 44/100

(Melibœe); 59/34; 268/54 (Melibe)

Menalcas/Menalchas 1; 3/48; 7/14ff.; 9/20; 28/1ff. (Minalcas); 40/0, 4, 102ff.; 47/6; 49/15; 53/2ff.; 59/38; 74/41; 76/8ff.; 77/19; 129/3ff.; 152/38 (Menalchus);

154/31, 82; 241/7ff.; 252/0, 1 ff.

Mersa 99/1ff.

Milo 1/47ff.

Mira 194/18ff.; 219/16, 175ff., 227;

$$
268 / 61
$$

Mirrha 53/3

Mirtillo 241/1ff.; 242/0, 23ff.

Moeris 196/1ff.

Moevius 221/79; 226/43ff.

Molorchus 97/13

Montanus 80/0, 9ff.; 242/0, 1ff. (Montano)

Mopsa 265/15ff.

Mopsus 21/1; 59/37; 207/1ff.

Morrell 41/1ff.

Motto 161/1ff.; 162/137ff.

Muridella 168/84ff.

Musella 211

Myrtil 220/13ff.

Naiis 7/46 (Nais); 167/1ff.

Neera 230/69

Nell 254/11ff.; 269/24

Nemincini 232/55

Nicias $2 / 0,1 \mathrm{ff}$.

Nico 49/1ff.

Nilkin 189/164

Nisa $252 / 3$

Nomaea $3 / 45$

Olcon $164 / 368$

Palinode 41/181; 64

Paloemon 106/26; 268/64ff.

Parnell 162/65

Parthenophe 91/29ff.

Parthenophill 91/1ff.

Pas 49/1ff.

Pastora 131/29; 238/3ff.; 271/46

Pastorella 44/45ff.; 45/2ff.

Pegg 228/38

Perigot 189/166; 268/24ff.; 271/44

(Perrigot)

Perin 138/0, 1ff.

Perkin 162/137ff. 
Persiu(s?) 240(B)

Peter, Sir 26/88

Philarchus 239/1ff.

Philarete 186/94ff.

Philemon 95/52

Philena 179/37ff.

Philisses 211

Phillida 114/0, 35; 116(B)/9; 31/1ff. (Phylida); 117/0, 6ff.; 119/15; 134/0, 1ff.; 152/5ff.; 155/0, 12ff.; $162 / 64 ; 270 / 30$

Phillis/Phyllis 9/37ff.; 57/20ff.; 58/1ff.; 59/1ff.; 60/1ff.; 71/32ff.; 74/57; 77/2ff.; 81/4ff.; 82/16; 89/2ff.; 106/40; 107/7ff.; 112/10ff.; 115/1ff.; 116/0, 9ff.; 117/6ff.; 118/20; 120/0, 1ff.; 138/143ff.; 143/11; 145/0, 1ff.; 148/102; 152/0, 5ff. (also as Phillis, 44); 154/34, 84ff.; 157/53; 160/111; 169/140, 173ff.; 170/3; 196/12ff.; 205/9ff.; 218/47; 269/29ff.; 271/43; 276/0, $70 \mathrm{fff}$.

Philorthus 239/0, 27ff.

Phoebe 80/11ff.

Phyllis: see Phillis

Piers 64/0, 5ff.; 139/0, 4ff.; 189/164

Poemenarcha 169/66ff.

Pollux 17/2ff.

Pratt 240A/2ff.

Proteus 219/110

Psyche 202/21ff.

Ralph 164/137; 215/59

Remond 189/49ff.

Ringde 83/3

Robin 18/1ff. (Robene/Robyne); $254 / 28$

Rock 164/141

Roget 185/0, 1ff.

Rollo 164/141

Rosalind 38/27ff.; 40/0, 44ff.; 45/103ff.; 161/232ff.. 228a; 162/62 (Rosalynde); 220/23
Rosania 273/0, $1 \mathrm{ff}$.

Rowland 96/102; 162/39, 193ff.

Rowly 189/166

Rye 240(A)/3ff.

Sapphus 206/26ff.; 208/5

Sarah 265/22

Silvan $189 / 165$

Silvia 260/1ff.

Silvio 242/0, 1ff.

Silvius 166/42ff.

Sirena 164/7ff.

Sophi(a) 132/27

Stella 43/73; 91/38ff.; 219/19, 117ff., $181 \mathrm{ff}$.

Strephon 51/1ff.; 154/25, 138ff. (Strephan); 268/0, $1 \mathrm{ff}$.

Susan 113/6; 228/38

Sylvanus $22 / 0$

Sylvia/Silvia 162/138; 195/0, 5ff.; 260/1ff.

Syrenus 22/0, 15ff.; 23/0

Tahah 215/4

Technis 206/1ff.; 208/1ff.

Teddy $189 / 165$

Thelgon 221(A)/28

Thenot 38/0, 1ff.; 138/0, 1ff.; 139/0, 1ff.; 146/1; 189/164; 220/0, 1ff.; 228/1ff.; 236/0; 268/38

Theorello 132/120

Therion 46/1ff.

Thestylis $7 / 10,43 ; 106 / 41-2$; 42/271ff., 296; 106/41ff.; 227/ 77

Thirsil 219/0, 46ff.; 220/14ff.; 221(A)/25ff.; 221(B)/7ff.; 221(C)/6ff.

Thirsis: see Thyrsis

Thomalin 41/1ff.; 219/18ff.

Thump (ram) 269/43

Thyrsilis 178/51

Thyrsis/Thirsis 58/25; 250/1ff.; 252/170; 262/25; 273/10; 277/5ff.; 154/32, 86 (Thersis); 
202/125ff.; 227/1ff.; 260/1ff.;

271/45 (Tyterus)

Tib $207 / 40$

Tim Crowd (fiddle?) 207/67

Tityrus/Tytirus/Titirus $4 / 1 ; 6 / 0$, 1; 32/65; 40/81; 42/2ff.; 59/29

(Titerus); 63/13; 74/40; 77/1ff.;

217/77; 226/1ff.; 227/6ff.;

268/83ff.

Tom 153/9; 164/133; 207/40; 212/0, 6ff.; 215/1; 238/0, 1ff. / 254/27; 269/0, 41ff.; 271/46

Tom T-239/297

Tom-Piper 184/83

Topas, Sir 161/128, 124a
Turnus 220/21ff.

Umber $17 / 19$

Urania 154/28, 126

Watt 62/1ff.

Wernocke 184/0, $1 \mathrm{ff}$.

Wilkin 169/137ff.

Will 153/199; 207/36; 238/0, 1ff.; $254 / 29 ; 271 / 46$

William 158/73ff.

William, Sweet 158/29ff.

Willy 138/89ff.; 183/1ff.; 184/0, 1ff.; 185/241; 205/1ff.

Winifred $155 / 91$

Wodenfride 136/0

(D) Mythological names and allusions

References under alternative names, sobriquets and paraphrases have been documented as far as possible. There are entries under generic (Dryades,

Fauns, Fairies) and allegorical (Aletheia) as well as individual names. Biblical names are in a separate list.

Poem number followed where appropriate by line number.

Achilles 5/80 ('Thetis' great son'); 8/43; 59/113 (his Pelian spear); $221 / 61$

Admetus 213/6

Adonis 9/18; 5/70; 106/44; 196/38

(Mirrhas child); 199/11; 204/89; 219/172

Aecidee: see Peleus

Aeolus 230/96 (Hippotades); 237/32, 168

Aesculapius 51/116

Aglaia 45/161; 65/37; 122/75ff. See also Graces.

Ajax 154/126

Alcinous 219/169

Aletheia (Alathea) 25/39

Amphion 7/23; 252/55ff.

Amphitrion 161/70, 66a
Apollo 5/26; 7/57 (Phoebus); 8/68; 9/21; 13/5 (Phoebus); 17/53; 35/4 (Phoebus); 38/73 (Phoebus); 39/33 (Phoebus); 40/68; 49/54ff., 120, 144 (Phoebus); 52/55 (Phoebus); 55/1; 57/59, 65; 58/3ff. (Phoebus), 80; 63/29 (Phoebus); 64/0.2ff. 61; 66/0, 1ff.; 67/7ff. (Phoebus); 71/34, 59 (Phoebus); 74/4 (Phoebus); 76/2 (Phoebus); 77/45; 80/8 (Phoebus); 86/8 (Phoebus); 92/55 (Phoebus); 97/5; 103/3; 106/60; 132/103; 138/99ff. (Phoebus), 120; 141/56ff., 80ff., 129, 157ff. (Phoebus); 147/38

(Sol); 148/117 (Phoebus); 
149/83ff (Phoebus); 154/10

(Phoebus); 159/51; 160/171a

(Phoebus); 161/71, 67a;

162/96 (Phoebus); 163B/29

(Phoebus); 165/23, 65ff.

(Phoebus), 86, 110; 167/133

(Delphian God); 184/161, 255

(Phoebus); 185/130; 186/86ff.

(Phoebus), 90; 188/2 (Phoebus);

$189 / 2 ; 190 / 172 ; 192 / 51 ; 193 / 3$

(Phoebus); 196/169 (Phoebus);

198/6; 199/10, 99 (Phoebus);

204/87; 213(A)/1ff.; 219/61ff.

(Phoebus), 165, 215ff., 246,

265; 220/26; 221(A)/75; 225/28;

228/149; 230/77 (Phoebus);

232/27 (Phoebus); 237/37ff.

(Phoebus), 232; 252/164

(Phoebus); 255/4 (Phoebus), 40 (Sol); 266/15 (Sol); 276/33

(Phoebus)

Argo (ship) 8/40

Argus 41/154

Ariadne 45/73; 162/219

Arion 202/192; 219/154

Astraea $8 / 5 ; 10 / 27 ; 65 / 1 ; 139 / 0,1 \mathrm{ff}$. (Elizabeth); 141/87 (Elizabeth)

Athena: see Minerva

Atlanta 154/100

Atropos 52/111

Aurora 5/44; 149/81; 159/57; 219/1ff., $152 ; 227 / 87 ; 237 / 29 ; 141 / 127$;

$196 / 171 ; 253 / 11$

Bacchus 10/105; 58/81; 161/8ff., 75, 71a; 191/11; 195/40, 45 (Lyæus); 219/170; 226/125

Bellona 55/34; 92/96; 161/7

Boreas 55/44; 141/33; 258/16; 276/75

Brutus (of Troy) 63/41; 65/3; 146/35ff. (Brute)

Calliope $5 / 73$; 8/67; 38/100; 40/57; $39 / 55 ; 230 / 58$

Camus: see Cam in list of place names
Caucus, King 131/24

Centaurs 45/76; 228/126

Cerberus 161/31

Ceres 58/81; 148/18, 107; 160/10; $185 / 163 ; 189 / 2,119 ; 195 / 39$; 196/49; 199/99; 237/168

Cerylus 5/42

Ceyx 5/40

Chamus: see Cam in list of place names

Chimaera 62/45

Chloris 38/122; 39/76 ('Chores')

Circe 199/84

Clarida 131/17

Clio 25/117. See also Muses.

Colossus 228/18

Comus 192/48

Cupid 3/67 (Love); 5/68 ('Cupids');

$31 / 89$; 32/12; 33/55; 44/61; 53/9;

60/2ff.; 67/59; 71/83; 72/13;

73/26ff.; 77/3, 50; 90/20; 77/3ff.;

$92 / 91 ; 116 / 12 ; 116(\mathrm{~B}) / 12$;

$118 / 17 ; 127 / 23 ; 128 / 13$;

140/0,39ff.; 140/12ff.; 147/37;

148/39,129; 149/31, 66; 151/83;

152/54; 154/51, 76; 155/49;

159/23; 160/97ff., 127ff., 127ff.;

189/353ff.; 196/136; 210/54;

217/109; 221(A)/36; 229/16;

257/22; 268/50 (Paphian

archer); 276/32

Cyclops 5/61; 161/97a; 199/84

Cyparissus 95/34

Daphne 66/0, 6ff.; 106/60; 148/118; 204/88; 219/66, 165, 207ff.

Demophoon 160/111

Diana 3/16, 19, 32, 66 (Phoebe); 8/7 (Lucina); 38/65 (Phoebe), 82 (Cynthia); 39/26ff. (Phoebe), 41 (Cynthia); 40/31 (Phoebe); 41/63 (Phoebe); 42/88ff.

(Cynthia=Elizabeth), 136, $160 \mathrm{ff}$. (Cynthia); 51/8; 61/8ff.; 64/67 
(Phoebe); 63/33 (Phoebe); 71/50; 80/11ff. (Phoebe); 90/8 (Cynthia); 91/56 (Delian Nymph); 92/40, 95 (Cinthia); 92/95 (Cinthia); 94/1 (Dian); $118 / 19 ; 126 / 53$ (Synthea); 134/45 (Cinthia); 148/49ff., $105 ; 151 / 85 ; 152 / 33 ; 154 / 12$ (Dyana), 44, 103ff. (Phoebe); 162/225 (Cynthia); 163(B)/88; 166/81 (Cynthia); 167/73 (Cynthia); 169/100; 176/21; 186/6 (Cynthia); 189/120; 196/85; 204/95; 219/150 (Dian), 250 (Delia); 237/238ff., 295 (Cinthia); 276/38 (Cynthia)

Dido $160 / 112 ; 176 / 17$ Dryades (Wood-Nymphs) 61/2; $121 / 59 ; 148 / 93$; 149/65; 154/55; $159 / 85 ; 163(\mathrm{~B}) / 5 ; 163(\mathrm{D}) / 22$; 166/79; 189/37; 191/10; 193/6, 36 ; 196/25; 201/11; 219/290; $252 / 21$. See also Napaeae.

Echo 5/55; 9/58; 49/134; 51/12; 52/31ff.; 82/10ff.; 91/8ff., 64; 106/119; 141/229; 186/106ff.; 190/121ff.; 193/42; 194/28; $202 / 20,171 ; 242 / 28$

Endimion $41 / 64 ; 134 / 45 ; 176 / 22$; 204/93-6; 219/251

'Erynnis' 217/2

Euphrosyne 45/160; 65/26. See also Graces.

Euronyme 45/155

Europa 228/116

Eurydice 5/130; 190/178

Eurynome 45/155

Fairies 40/25; 45/24, 114; 49/63; 86/39-40; 146/86; 147/30; $149 / 26 ; 157 / 8 ; 161 / 24,24 a$; $166 / 81 ; 167 / 74 ; 193 / 33 ; 199 / 92$; $202 / 11 ; 214 / 12 ; 218 / 40$

Fates: see Parcae
Fauns $5 / 28 ; 41 / 77 ; 49 / 63 ; 61 / 4 ; 91 / 17$; $104 / 1 ; 136 / 22 ; 149 / 47 ; 166 / 79$; $167 / 75 ; 191 / 18 ; 199 / 92 ; 203 / 15$; $230 / 36$

Faunus 57/60; 92/41, 70; 226/180

Feronia 183/95

Flora $35 / 2 ; 71 / 2,76 ; 86 / 27 ; 105 / 21$; $106 / 33 ; 121 / 45 ; 122 / 134 ; 131 / 9$; $136 / 6 ; 138 / 166 ; 141 / 28 ; 151 / 72$; $152 / 43 ; 160 / 7 ; 165 / 61 ; 218 / 11$; $228 / 161 ; 237 / 30,167$

Fortune 131/93

Furies 2/11; 217/2 (Erynnis); 52/111 (Atropos)

Galatea 2/0, 8, 19ff.; 5/59; 83/29; $106 / 39 ; 226 / 58 ; 227 / 4 f f$; 268/63ff.

Ganymede 97/1

Glaucus 219/219ff., 248ff.

Goblins 203/73

Graces 38/109; 39/63; 40/25; 45/41ff., $66,91-6,147-80,196-8 ; 65 / 9$, 39; 132/108; 136/34; 149/67; $164 / 170 ; 165 / 95 ; 174 / 23$; $189 / 241 ; 221 / 85 ; 228 / 153$. See also Aglaia, Euphrosyne, Thalia.

Gyges 225/27

Halcyon $5 / 40 ; 217 / 80$

Hamadryades 9/62; 132/16; 136/22; $166 / 79$

Hebe $127 / 20 ; 164 / 16$

Helen (of Troy) 3/1; 5/79 (Tyndarus' fair daughter); 92/89 (Tindaris); 184/156; 204/91

Hercules 5/124 (Alcides); 161/25ff.; 219/164 (Alcides); 228/148 (Alcides)

Hermes 51/10 (Mercury); 147/37; $183 / 100 ; 192 / 51 ; 193 / 7$

Hesperus 212/20

Hippotades: see Aeolus

Hobgoblin 256/22

Hyalus 106/44 
Hylas 252/65

Hymen 147/88; 149/69ff.; 257/22; $260 / 21$

Hyperion 149/1

Icarus 190/71

Ixion $190 / 73$

Janus $194 / 9 ; 221 / 118$

Jason 213(B)/8, 68ff.

Jove $8 / 58 ; 40 / 66 ; 45 / 154 ; 58 / 53$

(Jupiter); 63/11ff., 59; 64/117;

65/1, 8, 31; 83/51; 89/10; 97/5;

100/1ff.; 106/72ff.; 129/158;

132/33, 71; 147/37; 148/113ff.;

161/69, 65a; 162/211ff;

163C/72; 167/107ff.; 183/44ff.;

190/3; 202/19; 203/18 (Jupite)

r; 206/36; 217/41; 219/163ff.;

228/150; 230/16, 82; 232/5;

$237 / 240 ; 268 / 18$

Juno $100 / 25 ; 226 / 87 ; 237 / 240 ; 247 / 85$

Jupiter: see Jove

Lapiths 45/77

Latona 38/86; 39/46

Leander 190/100

Leda $148 / 114$

Leviathan $62 / 49$

Linus $8 / 66 ; 221(\mathrm{~A}) / 75$

Lucina: see Diana

Mars 9/44; 64/130; 67/63; 92/93; $163 \mathrm{C} / 72$

Maevius 221(A)/79; 226/43

Medusa 219/201

Meleager 154/99

Melpomene 25/117. See also Muses.

Memnon 5/43

Menelaus 5/80

Mercury 51/7; 94/0

Merlin 160/2; 161/63, 47a

Midas 221(A)/76; 224/4

Minerva 7/51ff.; 64/65; 100/26;

121/36; 148/106; 154/41ff.;

$169 / 100 ; 206 / 93,243 ; 219 /$

170
Mirrha 196/38

Molorchus 97/13

Momus 139/15

Muses 2/3; 5/8ff., 17 (Oeagrides), 66; 8/1ff.; 9/70ff. (Pierides); 10/31; 13/14; 15/49-50 (Parnasides); $20 / 36 ; 25 / 1 ; 38 / 41,102 ; 39 / 5$, 56; 40/28, 57ff.; 41/45/8; 42/19, 48; 46/6.1; 64/74; 77/47; 52/3ff.; 80/74; 91/10ff.; 95/11; 100/15ff.; $107 / 2 ; 112 / 7 ; 116 / 4 ; 118 / 7$; 121/1, 25, 34ff.; 122/135; 136/35; 138/38ff., 59, 71, 107; 139/2ff.; $147 / 31 ; 148 / 109 ; 160 / 173$; 161/12ff., 12aff.; 163(B)/1ff.; 163(D)/1ff.; 164/169; 165/6ff., 89ff.; 167/32ff., 130ff.; 178/60; 183/64; 184/54ff.186ff., 246; 185/0, 2ff., 90, 106ff., 130ff.; 186/91ff., 197; 190/69ff., 117; 201/16ff.; 191/14; 192/52; 194/1; 213/1ff., 25; 219/162ff.; 220/6; 221/8ff.; 221(A)/8ff., 104ff.; 226/25ff.; 228/156; 230/15, 58ff.; 237/57ff.; 243; 271/36. See also Clio, Melpomeme.

Naiads 9/10; 61/2; 106/36; 138/96; 166/149; 190/149; 193/6, 35; 196/27, 63ff.; 199/101; 203/37

Napaeae $92 / 73$

Narcissus 196/104

Nemesis $83 / 75$

Neptune 55/1; 163C/72; 166/158; 186/7; 203/110ff., 126ff.; 224/2; $230 / 90$

Nereids 166/158; 219/33, 289; 230/50, 99

Nereus 106/75; 190/150, 195

Niobe $38 / 87 ; 39 / 46 ; 237 / 197$

Nymphs 38/37, 122; 39/1, 71; 40/26; $41 / 80 ; 45 / 24,114 ; 49 / 66,73$; $51 / 2 ; 63 / 4,57 ; 86 / 13 ; 92 / 38$, $77 ; 106 / 31 ; 112 / 7 ; 116(\mathrm{~B}) / 30$; 
$121 / 59 ; 132 / 14 ; 134 / 40$;

$136 / 22 ; 138 / 59,96 ; 140 / 0$, 9ff.;

$147 / 31 ; 148 / 51,89 ; 152 / 22$;

163(D)/17; 165/89; 166/149;

$167 / 77 ; 174 / 23 ; 178 / 53 ; 179 / 15$;

$184 / 75 ; 185 / 84 ; 186 / 12,92$;

190/3; 193/35; 196/4ff.; 202/11;

203/40ff.; 214/2; 219/24ff.,

289ff.; 224/28; 230/50

\section{Oeager $5 / 17$}

Oenone 44/287; 71/72; 92/16ff.; $161 / 72 ; 176 / 20$

Oreades $147 / 29 ; 163 / 0,24 ; 196 / 27$

Orion $123 / 15 ; 228 / 135$

Orpheus 5/18, 123, 130; 8/65ff.

(Orph); 57/73ff.; 161/43, 51a; $190 / 177 ; 202 / 192 ; 214 / 16$; $228 / 93 ; 230 / 58$

Pales 57/59, 65; 183/94; 193/15; 194/30, 61; 199/100; 202/16; $227 / 22 ; 242 / 31$

Pallas: see Minerva

Pan 3/22, 38, 55; 5/56ff., 81; 7/31ff.; 8/69ff.; 9/26ff.; 38/51, 91; 39/14, 47; 40/30, 68; 41/49, 144, 179; 49/53, 141; 55/1ff.; 61/18ff.; 63/1; 77/15; 79/31; 91/5; 92/67; 94/7ff.; 106/24; 114/31; 131/2; $134 / 37 ; 136 / 25 ; 138 / 99 ; 141 / 7$; $143 / 7$; 145/21; 146/92; 148/19ff., $121 ; 152 / 23 ; 159 / 85 ; 168 / 29$; $169 / 100 ; 175 ; 183 / 45,93$; 186/90, 138; 191/11; 192/47; 193/1ff.; 194/14ff.; 195/22ff.; 196/185; 206/75ff.; 213/7ff.; 214/2ff.; 215/75; 218/38; 219/163ff., 211ff., 265, 278; 226/37ff., 94; 227/13ff.; 228/97, 171ff.; 239/67; 241/17; 242/31; 246/0, 16ff.; 254/3ff.; 262/1; 268/26ff.; 271/36; 275/2ff.; $277 / 37$

Panope 230/99
Parcae 138/171ff.; 152/27; 160/24; 184/77; 230/75 ('blind Furie')

Paris (of Troy) 3/1; 7/61; 17/45ff.; 41/145ff.; 44/286; 67/65; 71/71; 92/15ff.; 100/24; 176/19; $184 / 154 ; 204 / 91 ; 212 / 17$

Pegasus 228/124; 237/115

Peleus 45/158 (Aecidee); 221/61

Pelops 1/53

Penates 191/79

Penelope 169/83

Phaeton 63/35; 190/73ff.; 239/52

Phanes (Phanetas) 202/173

Phillis (lover of Demophoön) 160/111

Philomela (chiefly referring to the nightingale) $15 / 34 ; 52 / 61$; 63/37; 85/6; 92/61 (Dawlian Bird); 95/52 (Philomen); 103/5; 106/47; 107/6; 112/19ff.; 115/13; $142 / 12 ; 147 / 80 ; 163(\mathrm{~B}) / 60$; $164 / 199 ; 165 / 15 ; 203 / 23$; $214 / 13 ; 247 / 38 ; 252 / 14$

Piramus 190/200

Pluto 5/22, 125; 57/74; 106/6

Polyphemus $2 / 0,8,79 ; 5 / 61$

Pomona 62/8; 105/11; 219/169

Priam 67/68

Priapus 5/27 (Priapuses); 12/19

Proteus $1 / 52 ; 42 / 150 ; 219 / 110 \mathrm{ff}$., 217ff., 258

Pseustes (Sewstis) 25/39

Psyche 148/129

Saturn $8 / 5 ; 10 / 116 ; 63 / 40 ; 65 / 27$; $192 / 50$

Satyrs $51 / 3 ; 61 / 4 ; 86 / 14 ; 91 / 17 ; 92 / 67$; $106 / 33 ; 116(\mathrm{~B}) / 30 ; 132 / 17$; $136 / 22 ; 148 / 20,125 ; 149 / 25$; 166/79; 167; 185/38ff.; 191/17; $197 / 7 ; 203 / 15,65 ; 214 / 19$; $230 / 36$

Silenus 57/47

Sirens 59/101; 106/71; 228/139; 229

Sol: see Apollo 
Sybil 8/0.4ff. (of Cuma); 57/76; $161 / 61,45 \mathrm{a}$

Sylvans 41/78; 61/4; 104/1; 132/14, 77 (wood-gods); 166/75; 191/16; $196 / 61 ; 199 / 100$

Sylvanus/Silvanus 9/24; 12/20;61/4 (Silvani); 92/70; 131/16ff.; 148/127; 167/57; 192/47; $193 / 11 ; 194 / 22 ; 206 / 188$

Syrinx 38/50, 93; 39/13, 48; 49/57ff.; 61/1ff.; 94/1ff.; 148/122; 131/5

Tantalus $103 / 2$

Terpander $83 / 9$

Tethys $141 / 168$

Thalia 45/161, 65/39. See also Graces.

Theseus $45 / 75 ; 161 / 29 ; 228 / 148$

Thetis $5 / 80 ; 45 / 158 ; 106 / 75 ; 141 / 168$ (Tethis); 149/2; 159/81; 186/8; 190/ 1ff., 82 (Achilles mother); $201 / 64 ; 219 / 230 ; 276 / 42$

Thisbe 160/109

Thoosa $2 / 26$

Titan (Sun) 41/59; 71/64; 105/9; $129 / 135 ; 134 / 2 ; 151 / 41 ; 199 / 54$

Tithonus 219/1ff.

Triton 42/147; 203/115; 230/89ff. ('the herald of the sea')

Trophonius 201/50
Turnus 220/21

Tyndarus 5/79; 92/89

Typhis $8 / 40$

Ulysses $5 / 124 ; 154 / 126$

Venus $2 / 16$; 3/15, 16, 60, 67; 5/69; 25/130; 43/74; 45/37ff., 92ff., $148 ; 51 / 9 ; 58 / 19,32$ (Cytheræa); 60/1ff.; 61/7 (Cytherea); 72/11; 77/50, 58; 67/62; 71/60; $73 / 18 ; 76 / 51 ; 77 / 50 ; 100 / 25$; $105 / 30 ; 116 / 11 ; 116(\mathrm{~B}) / 11$; $127 / 24 ; 134 / 48 ; 148 / 17,35$, 101,$111 ; 149 / 27,68 ; 151 / 84$; 159/50, 74; 160/101ff., 128ff., 128aff.; 152/49ff., 59 (Citharia); 61/7 (Cytheræa); 154/46ff. (Citharea); 169/ 99 (Cytherea); 184/155; 196/37 (sea-borne queene); 204/90 (bewtyes queene); 219/171ff., 252; 226/186ff.; 227/60; 276/41

Vera $160 / 9$

Vesper 145/54

Vesta $105 / 20 ; 144 / 7 ; 247 / 158$

Vulcan $8 / 75 ; 161 / 101$

Zephyrus 92/82; 141/33; 199/29 (Zephires); 203/12; 255/5 (Zephyrs)

(E) Biblical names and allusions

Poem number followed where appropriate by line number.

Aaron 41/161

Abel 161/175; 213(A)/42 ff.

Abimelech 213/62

Abraham (Abram) 100/17; 213(A)/52ff.

Adam 16/6ff.; 40/10; 213(A)/43; $237 / 330$

Amos 213/119

Antichrist 239/321

Apostles 180/35
Baal 239/325

Belial 239/323

Cain 161/176

Christ: see Jesus

Dan 41/51

David 100/29; 133/41; 213/103 ff., $147 ; 221(\mathrm{~B}) / 23 ; 249 / 50$

Elias 237/70

Eve 16/6 ff.; 237/330

God/Lord 16/7; 17/21; 25/120; 
26/94 ff.; 35/53ff.; 41/9ff.;

67/106; 72/13; 87/1; 100/11,

37 (Jehovah); 110/1; 111/1ff.; 122/139; 187/1ff.; 188/6, 13;

247/19; 249/73; 254/32ff.

Goliath $100 / 31 ; 213 / 108$

Ishaye: see Jesse

Jacob 35/75; 41/144 (? 'the Mighty

Pan'); 183/34; 213/77

Jehovah 100/37

Jesse (Ishaye) 213/103

Jesus Christ 8/Argument; 16/13;

17/57; 18/93; 35/73 ('Juda's

Courageous Lion'); 100/44ff.;

$111 / 36 ; 133 / 33 ; 138 ; 178$;

180/52ff.; 202/51; 249/50;

255/73; 28/2; 133/33 ('Greatest

Shepheard'); 41/49 ('Great God

Pan')

Jethro 213/102

Jezebel 228/53

Job 213(A)/127; 202/32
Joseph 213(A)/91; 249/116

Laban 213/81ff.

Leah 213/83ff.

Lot 100/17; 213(A)/71 ff.

Lucifer: see Satan

Luke, St 213(A)/132

Magi 17/68

Mary 249/116; 271/25

Moses 17/49-52; 41/157; 100/36; 213/97 ff.

Nimrod 161/51

Peter, St 230/109 ('Pilot of the Galilean lake')

Philistine 35/76

Pontius Pilate 180/71 ('the Judg')

Rachel 213/83ff.

Rechab 202/32

Satan 110/12; 161/86, 82a (Lucifer); 221(B)/5 (Lucifer); 239/327

Saul 213/110

Solomon 202/139 (Salomon)

(F) Historical and other personal names and allusions

References under alternative names, sobriquets and paraphrases have been documented as far as possible. Biblical names are in a separate list. Names of possibly historical persons better known from classical myth or legend (e.g., characters in Homer) have been listed under 'Mythological Names'. The names of poets represented in this volume have been included only where occurring in works not their own.

Poem number followed where appropriate by line number.

Abdolonymus 225/13

Adam Bell: see Bell, Adam

Adelbrit (Adelbright), King 56/3ff.

Aglaus 225/23ff.

Alcaeus 5/90

Alcock, John 27/16ff.

Alexander (Emperor) 54/32; 161/41, $49 a ; 226 / 135$

Alexander, Anthony 199
Alexander, William 197; 198; 201/28

Alfred, King 153/5ff.

Anacreon $77 / 74$

Antony, Mark 9/0

Archilochus 5/92

Argentile 56/10ff.

Ariosto, Lodovico 190/125

Aristotle 206/10; 226/135ff.

('Shepherd of Stagira') 
Arthur, King 186/36

Astraea 10/27

Aubrey, Mary 273

Augustine, St 8/0

Augustus 6/0, 8/0, 15; 77/75ff.;

$217 / 78 ; 221(\mathrm{~A}) / 117$

Aurora 5/44; 149/81; 159/57; 219/1ff.; 227/87; 237/29; 141/127

Aylmer, John 41/0 (Morrell)

Aytoun, Robert 201/27

Barrow, Henry 239/250

Bavius 221(A)/78; 226/42

Beaumont, Francis 268/99

Beckett, Thomas 26/117

Bell, Adam 62/0, 34ff.

Bernières, Charles de 203/141 (Alcidon)

Bion 5

Brathwait, Richard 206 (Technis)

Brome, Alexander 271/0

Brooke, Christopher 183 (Cutty); 185/241 (Cutty)

Browne, Robert 239/228

Browne, William (Willy) 183; 184; 185/81-4, 241

Buckingham, Duke of 212/6ff. (Tom)

Cartwright, Thomas (?Thomalin) 41/1ff.

Cecil, Catherine 237/237ff.

Charles I 194; 212; 221(A)/117;

226/37ff.; 228/97; 241; 242; 250; $259 ; 261 / 27 ; 272 ; 277$

Charles II 250/135; 271

Cinnatus 12/40

Cleonymus 98/1

Cleopatra 160/110

Constantine 146/44

Curan 56/19

Cyrus 213(A)/139; 228/132; 237/279

Davies, John, of Hereford 184

(Wernock)

Davison, William 141
Devereux, Penelope 43/0, 73 (?Stella); $91 / 38$

Devereux, Robert, Earl of Essex 64/

Dedication; 129/74ff.; 221/92

Diogenes 54/31

Dodoens, Rembert 163B/221

Dover, John 228/172ff.

Drake, Francis 64/130

Drayton, Michael 96/102 (Rowland); 162/39, 193 ff. (Rowland)

Drummond, William 198 (Damon); 201 (Damon)

Du Bartas, Joachim 190/127ff.

Edelsie (Edell), King 56/3ff., 117

Edgar, King 163(A)/2

Elizabeth, Princess (daughter of James I) 185/43 (Thame)

Elizabeth, Queen 38/0, 34ff. (Eliza); 42/40ff.; 45/210 (Gloriana); 48 (Cynthia); 55; 63 (Eliza); 64/51, 58, 69, 155; 65 (Astraea); 66/36; 91/49ff.; 130; 136 (Cynthia); 139 (Astraea); 141/87, $111 \mathrm{ff}$. (Astraea); 142 (Cynthia); 146; 190/85 (Fayerie Queene), 195 (Nereus Queene); 221(A)/87 (Glorian)

Epimenides 5/97

Essex, Earl of: see Devereux, Robert

Eusebius 8/0

Fane, Mildmay 244/0

Ferrar, William 185 (Alexis)

Fletcher, Giles, the Elder 221/28 (Thelgon)

Fletcher, John 268/99; 174/0

Fletcher, Phineas 178/51 (Thyrsilis/ Thirsil); 219; 221(A)/25ff., 221(B)/7ff.

Frederick V, Count Palatine 185/43 (Rhine)

Gallus, Gaius Cornelius (Gallo) 9/0, 1ff.

Gamage, Barbara 191/19 
Garnier, Robert 190/135

Gerard, John 163B/222

Grindal, Edmund (Algrind) 41/126ff., 213ff.

Gustavus Adolphus 217/13

Helen, Saint 163(A)/25

Hengest 56/132

Henrietta Maria, Queen 194/18ff. (Mira)

Henry, Prince of Wales 147; 185/42; $191 / 77$

Henry VIII 62/93

Herbert, Mary, Countess of Pembroke 58/99

(Pembrokiana); 169/66 (Pœmenarcha)

Hesiod 5/88

Homer 5/72

Hood, Robin: see Robin Hood

Horace (Quintus Horatius Flaccus) 25/85; 232/1 (Flaccus)

James I 146; 185/42 (Pan); 191/76; 192/23; 194.14ff. (Pan); 212/17ff. (Pan), 37

James V of Scotland 212/37

John, Little See Little John

Jonson, Ben 226; 237/107; 268/100

Killigrew, Thomas(?) 238 (Tom)

Lactantius $8 / 0$

Lactantius Placidus(?) 25/37

Lawes, Henry 273/0

Little John 62/0, 34ff.; 156/96, $97 \mathrm{ff}$.

Livy 223/18

Ludwall, King 163A/2

Luke, St 213(A)/132

Machiavelli, Niccolò 223/18

Maecenas 184/65

Maevius 221(A)/79; 226/43

Mantuan (Baptista Spagnuoli) 25/33

Marot, Clément 190/135

Maxwell, Diana 237/238

Moray, Robert ('noble Murrey')

252/116
Morton, John, Bishop of Ely 27/5

Murray, William, Earl of Dysart(?)

(Will) 238

Nicias 2/1

Norreys, John 64/130

Oldisworth, Michael 215/0

Ovid (Publius Ovidius Naso)

77/41ff.; 190/122 (Naso); 213/4

Owen, Anne 273

Paul, St 204/168

Peter, St 230/109ff.

Petrarch (Petrarca, Francesco) 25/35; 190/125

Pharaoh 100/39

Philetas 5/99

Pindar 5/89

Plato 206/10

Plutarch 98/2

Pollio, Gaius Asinius 8/0, 1, $10 \mathrm{ff}$.

Ralegh, Walter 42/66 ('Shepherd of the Ocean'); 142 (Ocean)

Randolph, Thomas 226 (Damon)

Remus 10/113

Robarts, Margaret 221/38 (Gemma)

Robin Hood 62/0, 33ff.; 156/0, 4ff.

Romulus 213(A)/137

Ronsard, Pierre de 190/135

Salonius 8/0, $3 \mathrm{ff}$.

Sappho 5/93

Shakespeare, William 268/98

Sidney, Robert 191/50ff

Sidney, Philip 43 (Astrophel);

53 (Philisides); 64/64

(Philisides); 91 (Astrophil); 96/100 (Astrophel); 114/title (Astrophel, doubtfully Sidney); 138 (Willy)

Solomon (Salomon) 231/0

Soloninus $8 / 0$

Spenser, Edmund (alluded to) 190/185ff.; as Colin or Colin Clout, 38/0, 21, 154; 40; 42; 45/103ff.; 91/48; 96/99; 
138/41ff., 43, 86, 135, 204;

168/6ff; 190/86-8, 170ff.;

220/10ff.; 221(A)/85ff.

Stanley, James, Earl of Derby 261/14

Strangways 109/1

Tamburlaine 161/172, 168a;

213(A)/144

Tasso, Torquato $190 / 126$

Terpander $83 / 9$

Theocritus 5/100; 25/19

Tifernate, Gregorio (Umber) 17/19

Tilly: see Tserclaes

Tomkins, John 219 (Thomalin)

Tserclaes, John, Count of Tilly $217 / 18$

Virgil 25/27; 161/40, 40a (Maro);
190/120 (Maro); 217/76; 220/18-21; 221(A)/73 (Maro); $225 / 11 ; 228 / 94$

Volumnius 9/0, 2

Walsingham, Frances, Countess of Essex 43/Dedication, 73 (?Stella)

Watson, Thomas 96/101

Wenman, Lady Agnes 169/74 ('great Lady of the house of Thame')

Westminster, Roger, Prior of Ely $27 / 40$

Wilcox, Thomas (? Thomalin) 41/1ff.

Wither, George 185 (Roget); 186 (Philarete)

Wroth, Robert 192

(G) Place names (geographical and mythological)

Places are entered under their current names, except where well-established classical versions exist. Archaic, poetic or other variants are given in parentheses.

Poem number followed where appropriate by line number.

Achernus 161/32

Acheron 106/6; 161/32

Acidale 45/36

Adriatic Sea 190/108

Aegean Sea 190/102

Aesacus 92/39

Aetna 2/48; 5/128; 104/9

Africa (Roman province) 6/65

Aganippe 9/12; 148/142; 184/39ff.

Albion 65/52; 183/28; 190/169; 199/58

Allo 42/188

Alpheus 230/132

Alps 9/47; 51/67; 190/113; 228/160

Anglesey: see Mona

Antaprium: see Boarstall

Aonia 9/12

Arabia 10/11; 195/17

Aracynthus 7/24
Aran Fawddwy (Raran) 163(A)/17

Araris 6/62

Arcadia/Arcady 8/68ff.; 9/26ff.; 43/19; 49/52; 60/1; 61/1-3; 64/61; 91/3ff.; 96/69; 105; 106/20ff., 65, 79ff.; 131/1; 134/37; 137/2ff.; 148/147; 157/1ff.; 158/0; 186/69; 212/2ff.; 225/44ff.; 228/10; 237/114; 246/18; 250/21, 155; $262 / 1 ; 268 / 96$

Arden 43/114; 161/123, 119a; 163(B)/1ff.; 186/69

Arethusa 5/10, 78; 9/1;230/85

Arle 186/1

Arlesford 186/2 (Arlesford Pond), 33

Arlo 43/114

Ascra 5/88

Asia 190/104 
Aska Loua (Esk?) 196/18

Assyria 10/10; 17/39

Athens (for Oxford) 206/14ff., 268

Atlantic 199/39

Avernus 57/71; 106/9

Babylon 160/109; 228/60

Ballahoura: see Mole

Bayona 230/162

Bellerium: see Land's End

Bezdan 232/55

Bethlehem 100/44; 133/36; 241/22; 249/50ff. (Royal David's city); 251/21, 44

Black Sea: see Pontus

Boarstall 169/82

Bodotria: see Forth

Boeotia 5/89

Bohemia 217/17

Brenitia 56/132

Britain 6/67 (Britannia); 55/64; 163B/13; 164/281 (Brittany); 186/96 (Britany); 190/146 (Britany); 239/352

Brittany ('Armorick deepes') 190/142

Cadair Idris (Cadoridric) 163(A)/17

Caister (Cayastrus) 57/51

Cam 178/61 (Chamus); 219/48, 282 (Chame); 221(A)/13, 54

(Chamus); 226/129ff.; 230/103

Cambria: see Wales

Cambridge 62/67

Canaan 41/142

Cank 163C/39

Carthage 160/112

Caucasus 89/12; 95/5

Ceos 5/91

Chamus: see Cam

Cherwell 262/4

Chester 230/0

Colchis (Colchos) 213(B)/68

'Cornish Isles' 146/67

Cotswold 162/58; 163(C)/3ff.; 184/30; 228/101ff., 146ff.; 162/58
Cydonia(ns) 5/97. 9/59

Cytheron (Cythera) 45/42; 228/154

Dalmatia $8 / 0$

Darwin (river) 164/298

Denmark 56/19 (Danske); 212/40

Deva (Dee) 230/55

Diria 56/3

Dove (river) 161/148, 144a; 164/298; $276 / 26$

Dovan (Doven) 199/125

Dunbar 277/39

Edghill 277/39

Egypt 8/23; 9/0;100/39; 213/98

El Dorado 202/159

Ely 27/31, 54

Elysium 60/19; 91/40; 201/52 (Elysian fields); and as 'Elizium', 165/4ff.; 166/21; 167/0, 48; 183/86; 231/1 (Elyzium)

England 56/128; 65/44; 146/14ff.; 228/9ff.; 259/6, 30; 261/1; 272/1

English Channel (?'British Sea’) $190 / 152$

Esk 37/1; 196/18(?); 201/43, 62

Essex 163(D)

Ethiopia 9/67

Etna 5/128; 14/26

Etruria(ns) 10/112

Eubonia (Isle of Man) 261/4

Europe 190/104

Evesham 163(C)

Forth/Firth of Forth 198/7

(Bodotria); 199/125; 201/41

France 212/40;217/9;223/21

Funchin 42/187

Germany 223/14

Greece 17/54; 212/29; 213(B)/68; 228/11, 152

Haemony: see Thessalia

Hales 5/98

Hatfield 237

Hatfield Forest 163(D)/23ff.

Hawthornden 201/46 
Hebrides 230/156

Hebrus 9/65; 230/63

Helicon 20/37; 38/42; 39/6; 95/10; $148 / 142 ; 161 / 10 ; 184 / 42 f f .$, $80 ; 185 / 108 ; 190 / 117 ; 201 / 46$; $230 / 16$

Hellespont 190/99, 106

Herbert's Way 163(A)/25

Heptaphonos 202/171

Hermus 132/34ff.

'Hesperie' 206/61

Hibernia: see Ireland

Hippocrene 5/77 ('the Pegasean One'); 148/142; 230/15

Holland 217/5

Humber 164/324

Hybla (Hibla) 80/117

Ida, Mount 17/46; 92/17, 87; 41/57, $146 ; 100 / 24 ; 134 / 44 ; 161 / 71$, $67 \mathrm{a} ; 184 / 153 ; 228 / 158$

India $195 / 15 ; 199 / 104$ (Inde)

Indies $237 / 344$

Indus $232 / 28$

Ireland 146/68 (Hibernia); 188/12 (Hibernia); 189/12 (Hibernia); $261 / 1$

Irish Sea 230/0

Isca: see Usk

Isis $163(\mathrm{C}) / 65 ; 202 / 179 ; 252 / 113$

Israel 65/42; 100/33; 213/23ff.

Isthmia $228 / 151$

Itchen $186 / 1,35$

Ithaca $169 / 84$

Jerusalem 180/49; 251/17 (Salem)

Jordan $100 / 40 ; 178 / 30 ; 221 \mathrm{C} / 48$

Kent 41/44, 82; 161/149, 145a; 178/49; $221(\mathrm{~A}) / 113$

Kerig i Druidion 230/53

Kirkland 56/116

Ladon $61 / 24 ; 94 / 25 ; 96 / 62$

Lambeth 239/172

Land's End (Bellerus) 230/160

Lea $163(\mathrm{D}) / 2 \mathrm{ff}$.
Leominster (Lemster) 161/150, 146a; $163 \mathrm{C} / 21$

Lesbos 5/90; 230/63

Lestrigonia 99/2

Lethe $5 / 22$

Libanus 231/6

Limbo $161 / 36$

London 64/95; 65/2; 109/1; 163D/2ff.; $190 / 157 ; 228 / 108$

Hyde Park 274/66

Royal Exchange 274/54 (Exchange) Vauxhall Gardens 274/68 (Spring-Garden)

Lucrine 232/67

Lycaeum 193/56

Lycaeus 9/14 (Lices); 61/19

Maenalus 9/14ff., 55; 193/66; 212/19

Maas (Maes) 201/25

Man, Isle of 261

Mantua 6/0

Meander 201/42

Mediterranean ('Midland Sea') $190 / 107$

Medway 41/79ff.; 191/31; 221(A)/114, 130

Meles (Melus) 5/72

Merionethshire 163(A)

Mincius 201/44; 220/18; 230/86

Moesia 190/98

Mole (Ballahoura mountains) 42/57

Mona (Anglesey) 230/54

Moreland 162/138; 163C/39; 164/304 (Moorland)

Mulla (Awbeg) 42/59, 178

Mytilene 5/94

Nemancos (Namancos) 230/162

Nemea 228/151

Newton Court 153/22, 98

Nile 65/57

Northumberland 56/134

Nulam 109/9

Oaxis 6/66

Ochil (Ochell) 198/7 
Olivet, Mount 41/50

Olympus 57/72; 58/53ff.; 65/31; 106/1, $72 ; 161 / 34 ; 228 / 159 ; 239 / 53$

Orcades $146 / 67$

Ore (Ora) 201/43

Oxford 206/14ff., 268 ('Athens'); $235 / 32$

Pactolus 164/182; 224/3

Padus: see Po

Palestine 251/7

Paphos 92/48; 268/50

Parnassus $9 / 11 ; 38 / 41 ; 39 / 5 ; 40 / 28$, 70; 41/48; 167/30; 185/111; 221(A)/87; 226/5; 228/155

Paros (Parus) 5/92

Parthenius 9/57

Parthia(ns) 6/62, 9/59

Peak District (Peaks) 164/305

Peneus 219/247

Penechia (Peniche) 64/144

Penshurst 191/1ff.; 237/108

Pieria 161/10a

Pindus 9/11; 190/118

Plexippus 44/286

Po 92/18; 190/109ff. (Padus)

Pontus (Black Sea) 190/97

Psophis 225/46

Quinsai (Quinzey) 202/157

Rhine 9/47; 185/43; 201/25

Rome 6/20ff.; 10/61ff.; 41/183ff.; 64/141 (Rhemus town); 77/66ff.; 146/47ff.; 213(A)/137; 239/311, 334

Sabia/Sabines 10/111; 12/39

Salem 251/17

Salisbury Plain (Sarum's Plain) $163 \mathrm{C} / 19$

Salonius $8 / 0$

Salonoe $8 / 0$

Samos 5/96; 53/10

Sarum: see Salisbury

Saxham 234

Scotland 261/1
Scythia $6 / 66$

Serica 14/8; 221C/15 (Serian worms)

Severne 138/131; 163B/4

Sicily $1 / 0 ; 5 / 11 ; 7 / 21 ; 8 / 1 ; 9 / 4$

(Sicania), 51; 230/136

Sidon $221 \mathrm{C} / 18$

Sinai (Synah) 41/73

Sion $180 / 2 ; 239 / 351$

Sithonia $9 / 66$

Sodom 213(A)/73; 239/126

Somersetshire 153/21

Spain 64/93 (Western Coast); 212/31; $223 / 20 ; 228 / 67 ; 237 / 344$

Sparta $11 / 41$

St Bridget's Bower 41/43

St Helen's Way 163(A)/25

St Michael's Mount 41/41

Strymon 5/14

Styx 161/32, 36a

Syon $35 / 74$

Syracuse 5/100;25/20

Syria $8 / 30$

Tagus 164/182; 224/1;266/47

Tanais: see Tyne

Tavy, Tavistock 185/81

Tempe 10/48; 199/103; 228/15

Tenedos 190/98

Tethys $106 / 10$

Thame 169/74

Thames 41/83 (Themis); 57/1; $138 / 131 ; 184 / 29 ; 185 / 43,84$ (Thame); 190/157; 163C/65; 163D/20; 221(A)/130; 226/130; $228 / 108 ; 252 / 113$

Thessalia, Thessaly $17 / 55 ; 43 / 21$ (Haemony); 74/68; 76/17; $190 / 96$

Thrace $5 / 17 ; 8 / 65 ; 57 / 73 ; 190 / 177$; $214 / 14 ; 228 / 93$

Tiber 201/44

Tigris $6 / 63$

Trent 161/152, 148a; 163B/4; 164/165ff. 
Triopeum/Triopians 5/99

Troy $7 / 62 ; 8 / 42 ; 17 / 46 ; 64 / 85 ; 67 / 68$;

$92 / 85 ; 147 / 37$

Tyne 65/57 (?Tanais); 196/14ff.

Usk (Isca) 252/43ff.

Wales (Cambria) 146/68; 163A/23;

$163 \mathrm{C} / 39 ; 261 / 1$
Waltham Forest 163D/15ff.

Westminster $173 / 12$

Westwell Downs 235/1

Wight, Isle of $186 / 40$

Winchester 186/35-6

Xanthus 92/19ff. 